\title{
Virtual and Augmented Reality Technologies in Training Architecture and Civil Engineering Specialists *
}

\author{
Iurii Zgoda ${ }^{2[0000-0001-6714-500 X]}$, Alexey Semenov ${ }^{1[0000-0001-9490-7364]}$ \\ ${ }^{1}$ Saint Petersburg State University of Architecture and Civil Engineering, Saint-Petersburg, \\ 190005, Russia \\ yurii.zgoda@mail.ru \\ ${ }^{2}$ Saint Petersburg State University of Architecture and Civil Engineering, Saint-Petersburg, \\ 190005, Russia \\ sw. semenov@gmail.com
}

\begin{abstract}
This paper discusses the aspects of training architecture and civil engineering specialists using virtual and augmented reality technologies. It also addresses the issues of training specialists in software development for visualization of buildings and structures information models. The paper deals with the selection of optimal hardware and software for the most effective learning process and provides an overview of the most relevant areas of scientific research in this field.
\end{abstract}

Keywords: VR, AR, Building, Architecture, BIM.

\section{Introduction}

Virtual and augmented reality technologies are used in a wide variety of sectors, such as medicine, sports, scientific visualization, telecommunications, entertainment industry, as well as architecture and engineering.

Delgado et al. [1] highlight the main scenarios of using VR and AR, such as interaction with parties involved (to demonstrate various aspects of a project), visualization at the construction stage, assurance of industrial safety, and briefing on actions in emergency situations at a construction site.

The capabilities of these technologies are most fully utilized when used in BIM (Building Information Modeling). BIM is information modeling of buildings, which allows one to model not only the construction objects themselves but also their characteristics, as well as all possible changes in time [2].

Copyright (C) 2020 for this paper by its authors. Use permitted under Creative Commons License Attribution 4.0 International (CC BY 4.0).

* The research was supported by project "BIM-ICE - BIM Integration in Higher and Continuing Education" of "South-East Finland - Russia" CBC Programme 2014 - 2020. 
In practice, BIM technologies are used throughout the life cycle of a building or structure, i.e. during data collection, design, construction, operation, repair, and dismantling.

Thus, a BIM model includes both the geometric component and large volumes of metadata describing in detail each component of a building or structure being designed. Various scenarios for visualizing these metadata using VR and AR are available.

For example, a BIM model may contain information on building erection over a time period (4D-BIM) [3] making it possible to illustrate the stages of a building or structure construction project. A BIM model may include MEP components [4] allowing for volume rendering of heat carrier or air flows in the building.

A BIM model is a single repository of information about various sections of the designed building or structure, making it possible to automate the process of preparing visualizations using virtual and augmented reality technologies without the need for processing the BIM model in a 3D editor or writing program logic [5].

This paper addresses the application of virtual and augmented reality technologies within the educational process — both during training BIM specialists (which is an integral part of the educational process at the Saint Petersburg State University of Architecture and Civil Engineering) and specialists for development of interactive visualizations using VR and AR, trained by the Department of Information Technologies of the Saint Petersburg State University of Architecture and Civil Engineering.

The authors discuss the issue of choosing equipment for optimizing the educational process. They proved recommendations for the selection of visualization software and the development of interactive visualizations of BIM models. The paper also discusses the prospects for integration of virtual and augmented reality technologies and BIM modeling in Russia, and, in particular, at the Saint Petersburg State University of Architecture and Civil Engineering.

\section{Hardware equipment}

Currently, virtual reality equipment can be divided into desktop and standalone solutions. Desktop solutions are represented by Head-Mounted Displays to be connected to a computer. Oculus Rift S, HTC Vive Pro, Vive Cosmos Plus, and Microsoft Mixed Reality (MMR) HMDs are among the most popular hardware solutions in this sector. Oculus Quest and HTC Vive Focus Plus are the most relevant standalone solutions.

Desktop solutions enable a user to utilize the computing capabilities of a computer (which in most cases outperform the computing capabilities of a standalone HMD) for visualization. If interaction with BIM modeling software in virtual reality is required, desktop HMDs do not need any additional actions to transfer visualization to a virtual reality device. In case of using a standalone HMD, visualizations are streamed to the device or a BIM model in an intermediate format is transferred to the device and visualized using the computing capabilities of the HMD (a similar solution is described by Zgoda and Shumilov [5]).

A distinctive feature of a standalone HMDs is that they do not require any additional computing devices for running. Such devices can independently generate a stereo image 
and process user input using the built-in computing capacities. This means that their integration into the educational process does not require updating computer classrooms.

The disadvantage of such solutions is that their processing power is inferior to the processing power of a desktop HMD connected to a powerful computer, as well as the fact that initially, it was impossible to use a standalone HMD for debugging VR applications. Interactive visualization development environments such as Unity or Unreal Engine allow for real-time debugging of interactive visualizations in situations where a desktop HMD is connected to a computer, which significantly speeds up the implementation of new features and troubleshooting. Standalone HMDs are autonomous solutions. It means that for debugging we need to build an application in the APK installation format (most standalone HMDs are based on Android OS), transfer the APK file to the HMD, install the application, and run it. Only after that, it would be possible to debug the application being developed. This complicates and slows down the process of developing interactive visualizations for standalone devices.

Recently, it has become possible to connect virtual reality devices to a computer and use them as desktop HMDs. Oculus Quest is connected via a USB 3.0 cable, and HTC Vive Cosmos Plus is connected via Wi-Fi.

For this reason, the paper considers standalone devices as more universal. Desktop HMDs have slightly better performance characteristics, however, the impossibility to use them without connecting to a personal computer significantly limits their application.

HTC solutions (both standalone and desktop) represent solutions for the corporate sector, and therefore they are significantly more expensive compared with similar devices by Oculus. The eye-tracking feature available to HTC users can be used in the educational process [6], however, such a feature was not much needed in training courses at the Saint Petersburg State University of Architecture and Civil Engineering. Wireless connection to a computer for HTC devices is more convenient compared with using USB cables for Oculus devices, however, wireless transfer of video between a computer and an HMD may require upgrading the wireless network in the computer classrooms. In this case, one USB 3.0 port is enough to connect an HM to a computer via a USB cable. Moreover, wireless connection between a computer and an HMD for HTC is an additional feature requiring an appropriate HTC subscription.

Therefore, the simplicity of setting up and maintaining Oculus solutions, as well as the significantly lower price of these devices, make Oculus Quest an optimal solution for educational purposes.

Smartphones, tablets, Android emulators, or special AR glasses can be used for training architecture and civil engineering students using augmented reality technology (as well as training specialists in the development of AR applications). As for iOS devices, it is inexpedient to use those for training purposes due to the peculiarities of the Apple developer license.

At the time of writing this paper, only a very small number of augmented reality glasses could visualize 3D models using augmented reality. Most of these glasses only display some supporting information in the form of flat images or animations. According to the data sheet of one of the most popular AR development libraries - Vuforia [7], only three models of augmented reality glasses can be used with this library. 
Mixed reality solutions such as Magic Leap or Hololens provide significantly greater visualization compared with smartphones or tablets. However, their price is extremely high, and the demand for these devices in training architecture and civil engineering students is small (since these solutions are aimed at supplementing the real world with virtual objects, it is possible to make the most of them during building construction or operation but not at the design stage). The process of development regarding these devices in Unity and Unreal Engine development environments differs slightly from the development of mobile augmented reality applications using ARCore.

Thus, we suggest using Oculus Quest for training specialists in architecture, civil engineering, BIM and development of interactive visualizations using virtual reality technology. To ensure training using augmented reality technology during the educational process, any Android-based device supporting ARCore augmented reality technology will do.

The most important features for visualization using augmented reality are display quality and computing capabilities of the device used. Display quality affects the comfort of training. Device performance determines the complexity of BIM models visualized. As part of this study, a Samsung Galaxy S8 smartphone was tested to visualize BIM models, which demonstrated a consistently high frame rate during the visualization of BIM models designed by students when performing their individual tasks. Fig. 1 shows an example of the visualization of a BIM model using markerless augmented reality.

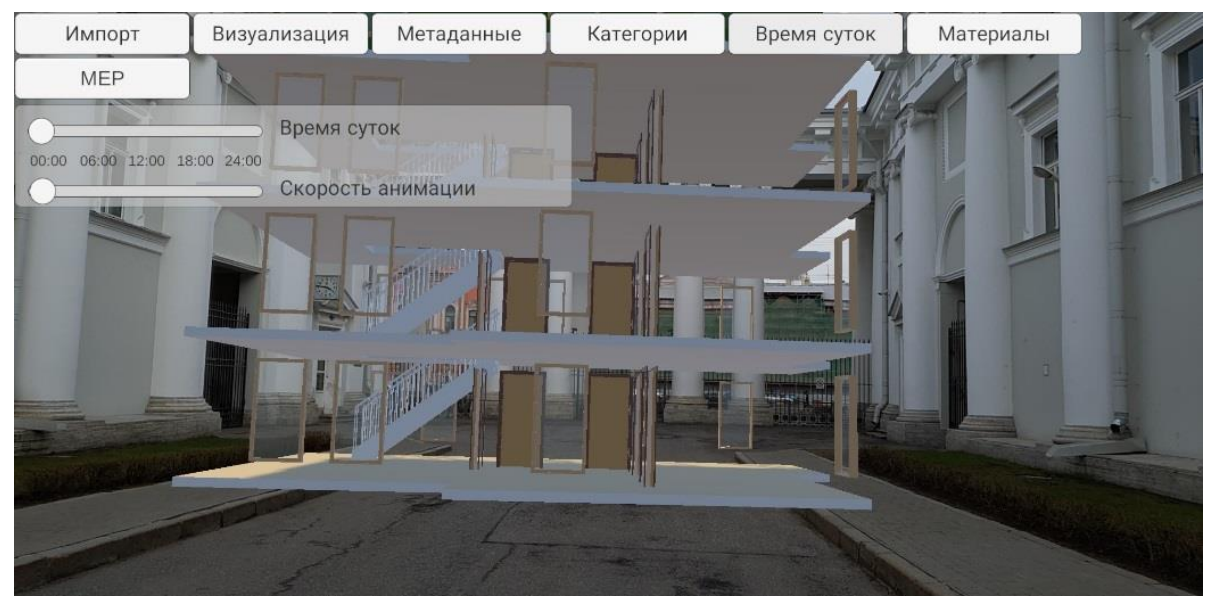

Fig. 1. Visualization of a BIM model using augmented reality

\section{BIM model visualization software}

Studies [8] show that the use of virtual and augmented reality technologies when training architecture and civil engineering students can significantly increase the effectiveness of training. Therefore, BIM models of buildings and structures designed by students need interactive visualization. 
Current solutions for the automated visualization of BIM models can be divided into two groups: those aimed only at visualization and those aimed at visualization using metadata. Solutions aimed at visualization provide a user with visualization quality up to photorealistic (Lumion, Twinmotion, etc.), and make it possible to study the designed building using virtual reality technology (however, without the support of augmented reality). These solutions are designed for desktop VR since they provide highly realistic visualization.

Solutions capable to display metadata allow users not only to visualize the geometric component of the designed building but also to display metadata for various elements of the model (Enscape, IrisVR, Insite VR, the domestic ConceptVR solution). Among the above solution, only Enscape is a desktop VR solution; the rest can be used with both standalone HMDs and desktop HMDs. However, their ability to work with metadata is limited to displaying metadata for specific building elements.

At the moment, only a limited number of solutions make it possible both to display metadata for specific building elements and also optimize visualization in accordance with these metadata (e.g. visualizing heat carrier flow movement in pipes based on MEP metadata [9]).

It should be noted that of all the above solutions only ConceptVR officially supports the domestic Renga BIM software. Other solutions can visualize Renga models only by using intermediate export formats. All the above solutions (except for Twinmotion having an educational license) are extremely expensive.

In this regard, we consider Twinmotion for photorealistic visualization of BIM models for students training. The Department of Information Technologies of the Saint Petersburg State University of Architecture and Civil Engineering proposed a hardware and software package [5] to visualize BIM models (including those designed with the domestic Renga software) using virtual and augmented reality technologies to study BIM models with metadata and MEP visualization.

\section{$4 \quad$ Interactive visualization development tools}

Currently, many software systems for the development of interactive visualizations are available. In certain situations, developing a solution from scratch can be effective. Nevertheless, the training course taught at the Saint Petersburg State University of Architecture and Civil Engineering currently lacks OpenGL, Vulkan or DirectX modules, therefore, students master tools for developing interactive visualizations during a corresponding course.

At the moment, the most common are Unity and Unreal Engine 4. In addition to those, there are other solutions, such as the domestic Unigine engine, the actively developing open-source Godot engine, CryEngine engine and its Amazon Lumberyard branch.

Unigine has become available to a wide range of users relatively recently and, therefore, requires a comprehensive detailed study to determine its applicability in the educational process. Godot is actively developing, which significantly complicates the teaching process. It is almost not used in a commercial environment, which makes it 
insufficiently relevant for specialist training. CryEngine and Lumberyard (as well as Unigine) currently do not provide development for mobile devices (which is necessary for studying augmented reality and standalone VR technologies). As a result, students need to master two separate engines during the course, which is impractical. Hence, this paper compares only Unity and Unreal Engine.

Currently, Unity and Unreal Engine have a similar component architecture, provide a high level of realistic visualization, support a number of intermediate formats, and are cross-platform (in terms of both the type of OS used for development and target platforms).

The Unity developer community is one of the largest and significantly outnumbers the similar Unreal Engine developer community. Moreover, Unity also offers its users much more comprehensive documentation. This greatly simplifies the search for information for students when they perform their individual tasks. The Unreal Engine documentation is quite detailed in a number of aspects. However, many highly specialized Unreal Engine components (such as procedural geometry generation, serialization, cross-platform file system API) are basically undocumented, and therefore information search for implementing any non-trivial functionality turns out to be more complicated compared with Unity.

Unity also provides a faster iteration process. The software products use C\# programming language, the code is executed in a virtual machine, and errors during program operation do not result in an abnormal end of development or interactive visualization. Moreover, compiling the code takes several seconds. Assembling a project as an executable file (for desktop systems) or an installer (for mobile devices) takes on average no more than a minute (for projects implemented by students as part of their term papers).

Unreal Engine offers users two development options. Using Blueprints visual programming language allows them to compile code in a fraction of a second. Besides, Blueprints is executed in a virtual machine, which isolates errors in the code and prevents emergency completion. Unfortunately, the use of visual programming means that although it is possible to implement something complicated in terms of writing software code, it is not too convenient.

Using C++ in Unreal Engine requires code coverage with a lot of auxiliary macros. There is no virtual machine, so any error (e.g. accessing an incorrect memory address) results in emergency completion of both visualization and the entire engine operation. The average compilation speed with minimal changes in the code is measured in tens of seconds. Assembling projects takes many minutes, which significantly slows down the educational process.

Cross-platform development is available in both engines, but Unreal Engine has significantly more restrictions. Given that at the moment there is no possibility of debugging augmented reality applications in an editor (both in Unity and Unreal Engine), development and debugging during independent work with Unreal Engine requires too much time, which negatively affects the effectiveness of the training process.

With a view to the above, Unity is a preferable tool when training specialists in the development of interactive visualizations in architecture and civil engineering, and, in particular, in the field of BIM. Unreal Engine offers a number of advantages, such as 
out-of-the-box photorealistic graphics (an example of visualizing a BIM model using Unreal Engine is shown in Fig. 2), the ability to use easier-to-learn visual programming, a large library of 3D modeling materials available to developers, and a number of features that are not available in Unity. In this regard, Unreal Engine 4 can be used to introduce architecture and civil engineering specialists to the development of interactive visualizations of BIM models.

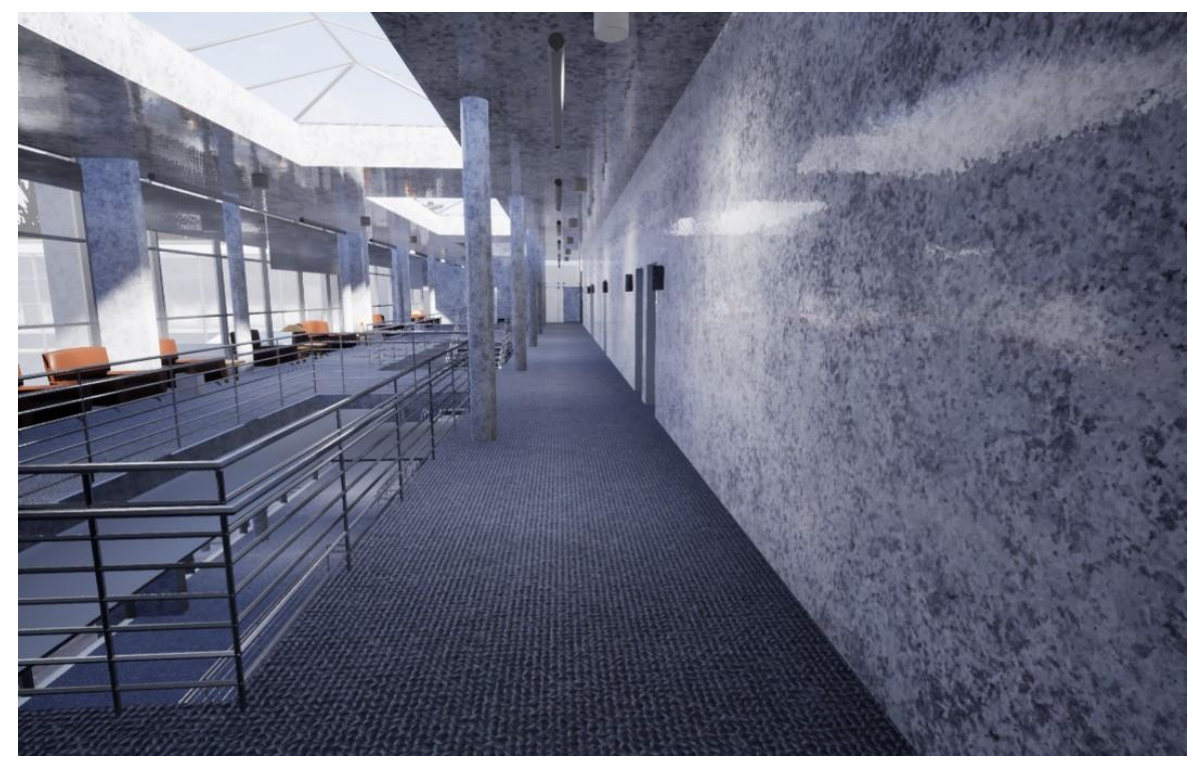

Fig. 2. Visualization of a BIM model using Unreal Engine 4

\section{Studies on the integration of VR/AR technologies and BIM}

BIM technologies, as well as virtual and augmented reality technologies, are actively developing. Therefore, this field of scientific knowledge in Russia has a large number of unexplored areas. It is being actively promoted at the Saint Petersburg State University of Architecture and Civil Engineering, where a number of studies are being carried out to expand the capabilities of virtual and augmented reality technologies in the BIM context.

In recent years, the Department of Information Technologies of the Saint Petersburg State University of Architecture and Civil Engineering has been actively studying the preparation of photorealistic visualizations of BIM models using virtual and augmented reality technologies [10].

A number of studies conducted at the Department are aimed at identifying new nonstandard and effective scenarios for using VR/AR in the BIM context. There are highly specialized papers related to the use of virtual reality for safety briefing [11], creation of interactive visualization with the display of energy efficiency parameters of buildings and apartment layouts [12]. More general issues related to the automated 
generation of interactive visualizations of BIM models (including MEP, see Fig. 3) using virtual and augmented reality are also considered [5]. Particular attention is paid to supporting domestic Renga BIM software.

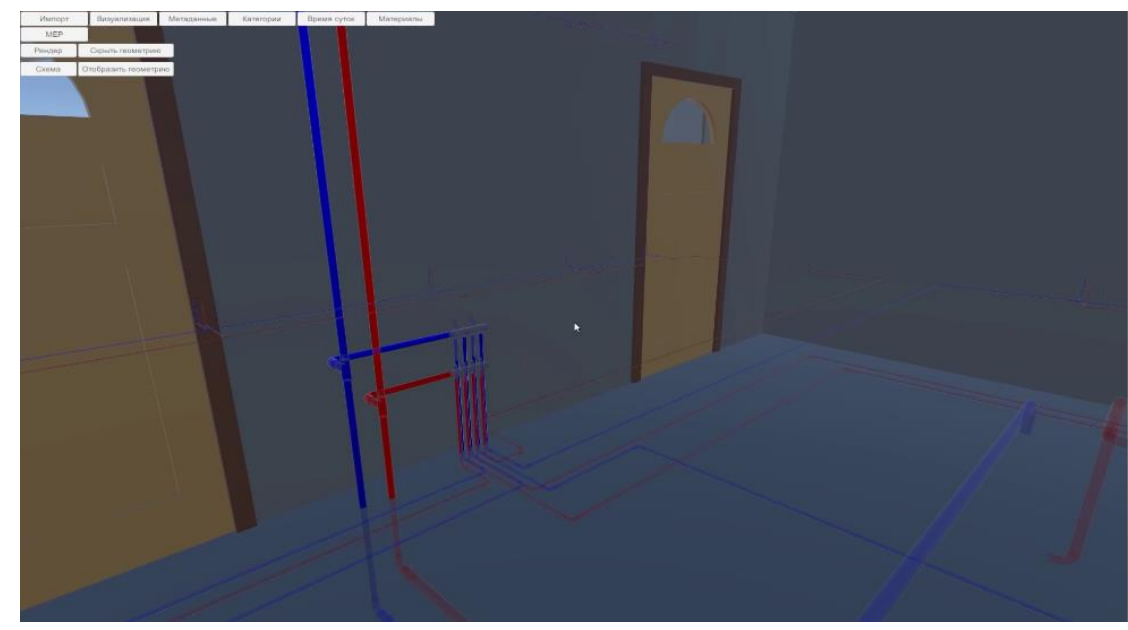

Fig. 3. Visualization of a BIM model with MEP display

\section{Conclusion}

In the course of the study, we performed the comparative analysis of the solutions for virtual and augmented reality technologies, their advantages and disadvantages in the context of training BIM and interactive visualization specialists. We also reviewed the issues of choosing software tools for visualizing BIM models and development environments for interactive visualizations, and described the most promising areas of research for this subject at the Saint Petersburg State University of Architecture and Civil Engineering.

We recommend Oculus Quest HMDs as training equipment with the use of virtual reality technologies because of their easy setup, accessibility and no need for highperformance computers. We also recommend Android smartphones with ARCore technology support as training equipment with the use of augmented reality technology, characterized by high computing power for real-time visualization of BIM models and high-quality display.

Unity and Unreal Engine interactive visualization development environments are recommended for training specialists in the development of interactive visualizations of buildings and structures. Unreal Engine offers visualization quality close to photorealism and visual programming tools that simplify the process of developing program logic for specialists without a profound knowledge of programming. Unity is currently an optimal development environment for training specialists with programming skills in high-level languages, for whom programming and creation of interactive components will be the main focus in the development environment. 


\section{$7 \quad$ Acknowledgments}

The research was supported by project "BIM-ICE - BIM Integration in Higher and Continuing Education" of "South-East Finland - Russia" CBC Programme 2014 2020.

\section{References}

1. Davila Delgado, M., Oyedele, L., Demian, P., Beach, T.: A research agenda for Augmented and Virtual Reality in Architecture, Engineering and Construction. 45101122 (2020). https://doi.org/10.1016/j.aei.2020.101122.

2. Milyutina, M.A.: Introduction of Building Information Modeling (BIM) Technologies in Construction. JPhCS, 1015(4), 042038, 4 pp. (2018).

3. Jupp, J. 4D BIM for environmental planning and management. Procedia engineering, 180, pp. 190-201 (2017).

4. Khanzode, A, Fischer, M, Reed, D. Benefits and lessons learned of implementing Building Virtual Design and Construction (VDC) technologies for coordination of Mechanical, Electrical, and Plumbing (MEP) systems on a large Healthcare project. Journal of Information Technology in Construction, 13, 19 pp (2008).

5. Zgoda, Iu.N., Shumilov, K.A.: Markerless Augmented Reality in BIM-Model Visualization. Proceedings of III International Conference "BIM in construction \& architecture", pp. 217 222 (2019). https://doi.org/10.23968/BIMAC.2020.028

6. Rahman, Y., Asish, S.M., Khokhar, A., Kulshreshth, A.K., Borst, C.W.: Gaze data visualizations for educational VR applications. Symposium on Spatial User Interaction (2019). https://doi.org/10.1145/3357251.3358752

7. Vuforia Engine for Digital Eyewear https://library.vuforia.com/content/vuforia-library/en/articles/Training/Vuforia-for-Digital-Eyewear.html. Last accessed 1 Jul 2020.

8. Sun, C., Wei, H., Diqiong, X.: Navigation modes, operation methods, observation scales and background options in UI design for high learning performance in VR-based architectural applications. Journal of Computational Design and Engineering 6.2, pp. 189-196 (2019). https://doi.org/10.1016/j.jcde.2018.05.006

9. Fuzor https://www.kalloctech.com/index.jsp. Last accessed: 01.07.20.

10. Zgoda, Iu.N., Semenov, A.A., Shumilov, K.A.: Development of photorealistic interactive BIM-model visualization with use of virtual and augmented reality technologies. New information technologies in architecture and civil engineering. Conference proceedings, pp. 24 (2018).

11. Shakshak, O.M., Subbotina, N.A., Tsaplin, V.V.: Interactive digital model as a modern teaching method. IV All-Russian Scientific and Practical Conference with international participation "Safety in construction": conference proceedings, pp. 32-40 (2019).

12. Shakshak, O.M., Evsikov, I.A.: Multifunctional VR application based on digital building model. Architecton: Proceedings of Higher Education, 4 (68), 11 pp. (2019). 